\title{
MICROSPECTROPHOTOMETRIC AND PSYCHOPHYSICAL RESULTS FOR THE SAME DEUTERANOPIC OBSERVER
}

\author{
J. D. MOLLON, J. K. BOWMAKER, H. J. A. DARTNALL and A. C. BIRD \\ (Cambridge/London/Brighton, U.K.)
}

\begin{abstract}
Microspectrophotometric measurements have been obtained for individual receptors from the enucleated eye of a known deuteranope. In a sample of 17 receptors we recorded 5 rods, 2 short-wave cones and 10 long-wave cones but no middle-wave cones. The results are concordant with psychophysical results for the patient's remaining eye.
\end{abstract}

\section{INTRODUCTION}

We have recently been able to secure microspectrophotometric measurements of retinal tissue from a patient known to be colour-deficient. Our microspectrophotometric results are limited, but we place them on record for two reasons. First, owing perhaps to his professional training, the patient is an extremely careful observer and thus it has been possible to complement the microspectrophotometric measurements with systematic visual measurements for the patient's remaining eye. Second, this is not only the first such case to be examined microspectrophotometrically, but could be the last for some time to come: in recent years, ophthalmologists have become increasingly selective in adopting enucleation as the preferred treatment for small ocular melanomas (Albert, 1982), particularly in the presence of good visual acuity.

The patient was aged 75 at the time of the operation. He has been aware of his colour deficiency since childhood. However, before his retirement he was the Chief Inspector of a large electronics company and did not find his colour deficiency a serious handicap; he carried a pocket Avometer with which to check resistances when occasion arose. He reports that his brother and his maternal grandfather (both deceased) were colour deficient. His right eye was enucleated at Moorfields Hospital in December, 1981. On the evening before his operation, the patient performed several clinical tests of colour vision with his left eye; his vision with his right eye was too poor to allow testing. He was able to visit Cambridge to make further psychophysical measurements in May, 1983. 
Table 1. Results of plate tests.

1. Ishihara Screening plates ( 9 th ed.):

Ishihara Classification plates (Nos. 22-25):

$18 / 18$ errors

$3 / 4$ deutan; 1 unclassified

2. SPP Screening plates: SPP Classification plates:

$10 / 10$ errors

$3 / 5$ deutan; 2 unclassified

3. Okuma Screening plates:

$7 / 7$ errors

Okuma Classification plates:

'Severe deutan' pattern

4. TCU 1 st edition ( 3 repetitions):

$27 / 30$ errors; 24 deutan, 3 protan

5. TCU 2nd edition:

9/10 errors; 8 deutan, 1 protan

6. Farnsworth tritan plate:

Pass

7. Birch tritan plate:

Pass

Note: CIE illuminant $C$ was used for all tests. Tests 1,3 and 4 were performed with left eye in December, 1981, before enucleation of contralateral eye; tests 2 and 5 were performed in May, 1983.

\section{TYPE OF COLOUR DEFICIENCY}

Table 1 summarises the patient's performance on a number of pseudoisochromatic and other booklet tests. All these tests were administered under CIE Illuminant C (Macbeth Easel lamp).

The tests are extremely consistent in classifying the patient as severely deutan. Three of the four protan responses on the two editions of the City University test were to the same plate (Plate 7 of the 1st Edition, Plate 5 of the 2nd), which is known to misclassify deuteranopes (Dain, 1980; Birch, this volume). The patient passes the Farnsworth tritan plate and an unpublished tritan plate kindly supplied by J. Birch.
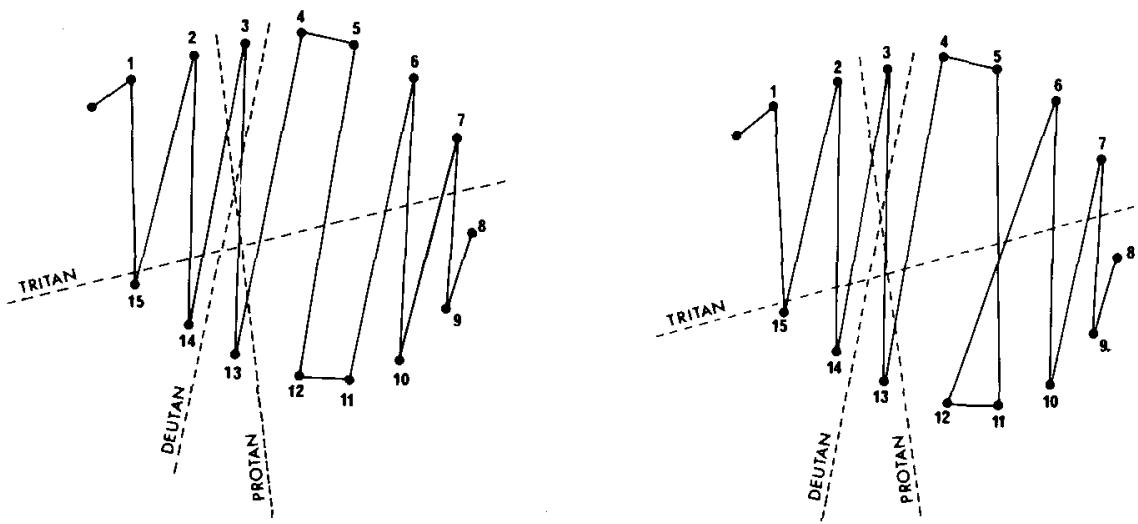

Fig. 1. Results on D15 test. Two successive arrangments are shown. 
Results for two successive arrangements of the D15 test (Fig. 1) show consistently deutan responses, the left-hand set of results corresponding exactly to the paradigmatic pattern given for deuteranopes in the report on colour vision testing by the Committee on Vision (1982). On the FarnsworthMunsell 100-Hue test the patient had a total error score of 220 on the first test and 305 on the second test. Mean results are shown in Fig. 2, lower right The errors show a classical deutan pattern.

On the Nagel anomaloscope (Model II), with primaries set at 541 and $666 \mathrm{~nm}$ to match $589 \mathrm{~nm}$, the patient was able, by adjusting the radiance of the yellow field, to match all red-green ratios set by the examiner.

During his visit to Cambridge in 1983, it proved possible to establish a spectral neutral point for the patient. By means of a computer-controlled Maxwellian-view optical system, isolated flashes subtending 1 degree of visual angle and lasting 1 second, were presented to his dark-adapted eye. For the purpose of estimating a neutral point, we adapted a randomised doublestaircase procedure of the kind used by Mollon and Polden (1977) to measure increment thresholds: by means of a monochromator with integral stepping motor (Bentham Instruments), the program made sequential adjustments of the wavelength of the flashes according to the patient's responses 'warm' or 'cold'. The step size was $1 \mathrm{~nm}$ and the two staircases began $20 \mathrm{~nm}$ apart (at 490 and $510 \mathrm{~nm}$ ); successive presentations were drawn randomly from one or other staircase. This procedure avoids the biases that may be introduced when the Method of Adjustment or the Method of Constant Stimuli are used to measure a neutral point (Hurvich and Jameson, 1974). Four independent runs gave a mean neutral point of $503 \mathrm{~nm}$ for our patient, the total range of the four estimates being $1.4 \mathrm{~nm}$.

In summary, all tests are consistent with the conclusion that the patient is a deuteranope.

\section{MICROSPECTROPHOTOMETRIC RESULTS}

Microspectrophotometric measurements were begun within 1 hour of enucleation. When the globe was opened, much of the retina was found to be detached and the foveolar region could not be identified. However, a number of samples were prepared from intact areas of retina, using procedures described in detail elsewhere (Knowles and Dartnall, 1977; Bowmaker, Dartnall and Mollon, 1980). The measuring beam of a computer-controlled Liebman microspectrophotometer was passed transversely through the outer segments of individual photoreceptors while a reference beam was passed through an adjacent clear space in the preparation. Absorbance spectra were obtained for a total of 17 cells. The transversely measured absorbances were low (mean values: 0.009 for long-wave cones, 0.011 for rods, and 0.02 for shortwave cones).

For each cell recorded, the wavelength of peak absorbance $\left(\lambda_{\max }\right)$ was estimated from the spectral position of the right-hand limb of the raw absorbance spectrum: each of 20 empirical absorbance values on the long. wavelength limb of the curve (corresponding to a $40 \mathrm{~nm}$ segment of the 
record and to percent absorbances in the range approximately 45-90\%) was referred to an appropriate template in order to obtain an estimate of the $\lambda_{\max }$. This operation amounts to finding where the standard template must be placed to give the percent absorbance value under consideration. Since it is now clear that absorbance spectra are not constant in shape when plotted against wavenumber (see, e.g., Ebrey and Honig, 1977; Knowles and Dartnall, 1977) different templates were used for different classes of receptor: for the short-wave cones, the frog 'green rod' template of Bowmaker (Knowles and Dartnall, 1977, p. 76); for the rods, the Dartnall template (ibid); and for the long-wave cones, a template based on our own measurements of macaque long-wave cones.

The distribution of $\lambda_{\max }$ values for the deutan is shown in Fig. 2 and can be compared with the distribution for a male colour-normal patient aged 56 , whose eye was examined by very similar procedures one month earlier. Of the 17 receptors recorded for our deuteranopic patient, 2 were short-wavelength cones, 5 were rods and 10 were long-wavelength cones. Our sample for this patient contained none of the middle-wave cones that can be seen in the distribution for the colour-normal patient (No. 8) and which are typically found to peak near $530 \mathrm{~nm}$ for human observers (Dartnall, Bowmaker and Mollon, 1983). The mean value of $\lambda_{\max }$ for the long-wave cones of the deuteranope $(558.4 \pm 4.4 \mathrm{~nm})$ corresponds closely to the value found by Dartnall et al. (1983) for colour-normal eyes. The mean $\lambda_{\max }$ for the two short-wave receptors was $427 \mathrm{~nm}$ and that for the rods was $499 \mathrm{~nm}$.

\section{SPECTRAL SENSITIVITY}

When the patient visited Cambridge in May, 1983, we attempted to estimate psychophysically the spectral sensitivity of his long-wave cones. Using a computer-controlled Maxwellian-view apparatus (Mollon and Polden, 1977), increment thresholds were measured for brief $(22 \mathrm{msec})$, small (4.8 minutes of visual angle) test flashes delivered to the centre of the foveola. These are conditions that are thought to minimise the contribution to detection of the short-wave cones and of the rods (see e.g. Willmer, 1949; Farnsworth, 1955; Mollon, 1982). The flashes were presented on either a long-wave $(637 \mathrm{~nm})$ or short-wave $(480 \mathrm{~nm})$ field, subtending $4 \mathrm{deg} 24 \mathrm{~min}$ and of a radiance chosen to raise the threshold by approximately $1 \log _{10}$ unit. At each of ten test wavelengths, thresholds were measured by means of a double randomised staircase procedure; the patient responded 'Yes' or 'No' by means of pushbuttons. A $2 \mathrm{~mm}$ artificial pupil was used and the patient rested on a chin-rest. Two sessions were devoted to each field wavelength and thresholds were measured for all test wavelengths in each session.

In Fig. 3 the filled circles show the psychophysical thresholds measured on the long-wave field and the unfilled circles show thresholds measured on the short-wave field. An important result is that there appears to be no systematic difference according to the wavelength of the field (unless at the shortest test wavelength, where contamination by the short-wave cones may perhaps occur in the presence of the $637 \mathrm{~nm}$ field): in both cases sensitivity 

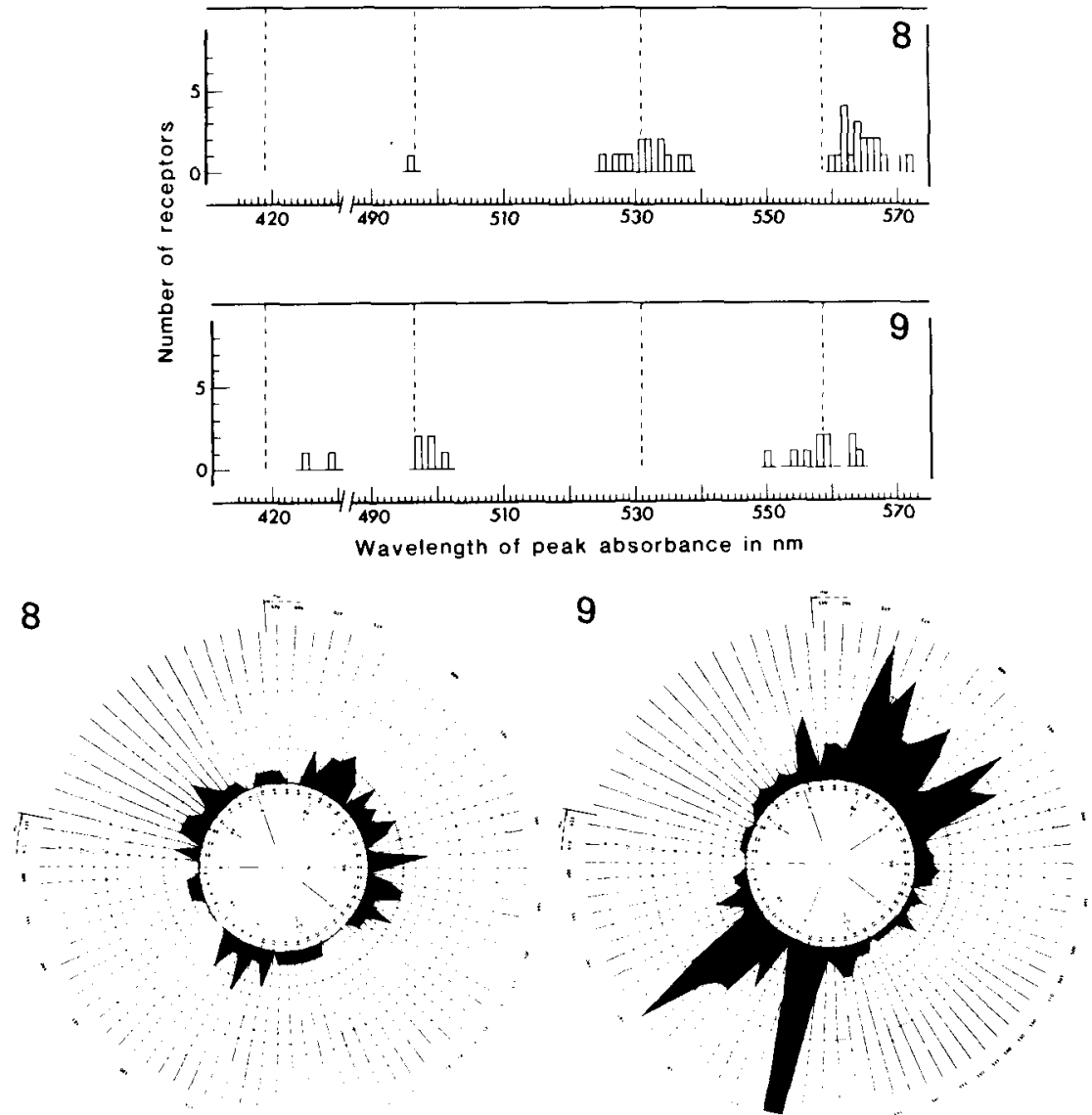

Fig. 2. Results for the deutan patient (identified as Patient 9), compared with those for a colour-normal observer (Patient 8 in our series). Below are shown each patients' error scores on the Farnsworth-Munsell 100-Hue test (means for two tests in each case) and above are shown the spectral distributions of recorded photoreceptors. The value plotted is the wavelength of peak sensitivity $\left(\lambda_{\max }\right)$ and the bin size is $1 \mathrm{~nm}$. The vertical dashed lines in the upper plots represent the mean $\lambda_{\max }$ values for the four classes of photoreceptor found in colour-normal human retinae (Dartnall et al., 1983). Note the absence of middle-wave cones in the sample from the deutan retina (Patient 9). In the colournormal retina (Patient No. 8) no short-wave receptors were found: this is not unusual, since these receptors appear to be rare in all human retinae, but their absence serves to emphasise that we cannot exclude the possibility of small numbers of middle-wave receptors in the deutan retina.

peaks in the region of $560 \mathrm{~nm}$. This is the result to be expected for a deuteranope. In the case of a normal observer, the long-wave cones would dominate test sensitivity on a short-wave field and the middle-wave cones would dominate on a long-wave field.

The solid line in Fig. 3 shows the psychophysical sensitivity that might be 


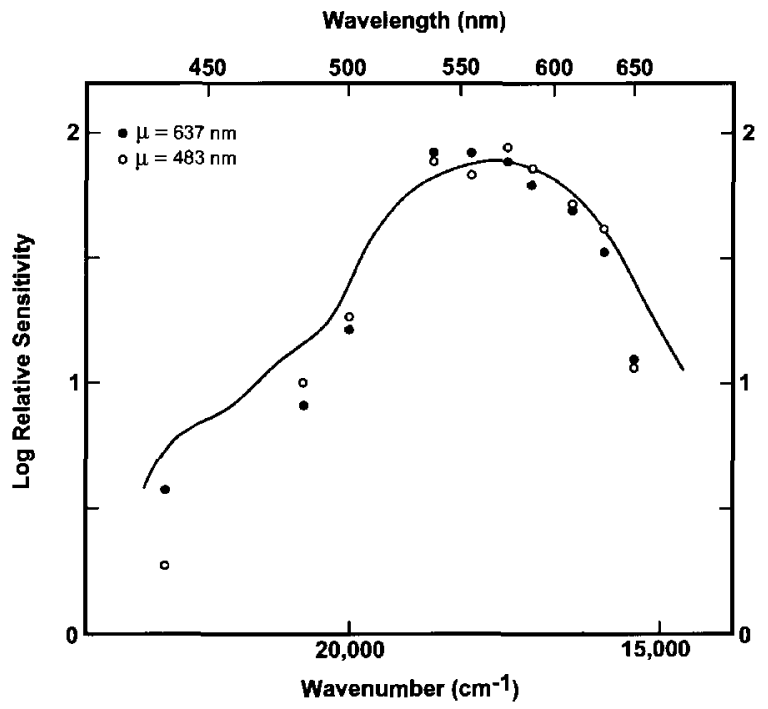

Fig. 3. Spectral sensitivity of the deutan observer estimated psychophysically and microspectrophotometrically. The symbols show increment thresholds for brief, small flashes presented on either a long-wave $\left(637 \mathrm{~nm} ; 8.73\right.$ quanta $\mathrm{sec}^{-1} \mathrm{deg}^{-2}$ ) or a short-wave field (483 nm; 8.68 quanta sec ${ }^{-1} \mathrm{deg}^{-2}$ ). The solid line shows corneal psychophysical sensitivity reconstructed from the mean absorbance spectrum for the patient's long-wave receptors; this reconstruction depends on several assumptions, which are discussed in the text.

predicted from the mean absorbance spectrun of the patient's microspectrophotometrically measured long-wave cones. We emphasize that it is necessary to make a number of assumptions in any reconstruction of corneal psychophysical sensitivity from measurements of the transverse absorbance of individual outer segments (Bowmaker et al., 1980); and in the present case the uncertainty is increased by the corrections needed to allow for agerelated changes in visual sensitivity. In order to estimate the absorptance of the patient's receptors we have assumed that his outer segments are effectively shorter than those of younger observers by $40 \%$ (J. Marshall, personal communication) and that the axial density is therefore about 0.3 . To correct for absorption by the macular pigment we have taken the values tabulated by Wyszecki and Stiles (1982, Table 2(2.4.6)). To estimate the spectral absorbance of the lens of our 77-year old observer we have taken the values given in Table 1(2.4.6) of Wyszecki and Stiles (1982), which are for young observers (approximately 20-30 years old); and have multiplied these values . by 2.04 , as is required by the formula given by Wooten and Werner (1980) for the relation between age and the density of the lens. Since the older observers in the study of Wooten and Werner would have had smaller pupils than the younger ones, we have adopted the conservative assumption that no additional correction is required for the fact that our psychophysical stimulus was confined to the centre of the pupil and thus the widest part of the lens. 
Given the uncertainty associated with the corrections that must be made in the reconstruction, and given the known factors that may contaminate microspectrophotometric measurements at short wavelengths, such as photoproducts, focusing error and wavelength-dependent scattering (MacNichol et al., 1983), the agreement in Fig. 3 is as satisfactory as one could expect. The psychophysical and microspectrophotometric functions peak in the same spectral region. It would be possible to improve the fit by assuming a higher lens density and a smaller self-screening effect; but to make further ad hoc adjustments would be to misrepresent the precision with which such comparisons can be made.

\section{CONCLUSIONS}

The present microspectrophotometric measurements are the first to be reported for a colour-deficient human observer. Like earlier objective measurements by reflection densitometry (Rushton, 1965; Alpern and Wake, 1977), our results are consistent with the classical 'loss' or 'reduction' hypothesis of deuteranopia (Voigt, 1781; Clerk Maxwell, 1855). Clearly, with a relatively small microspectrophotometric sample, we cannot exclude the possibility that a small number of middle-wave receptors were present in this deuteranopic retina. However, our results are inconsistent with the class of hypothesis that supposes that long- and middle-wave receptors are present in normal proportions in deuteranopic observers but that their signals are in some way fused at a post-receptoral level (e.g. Wright, 1967). In the sample of seven colour-normal eyes examined by Dartnall et al. (1983), there were 49 middle-wave cones and 69 long-wave cones. If we assume that these numbers roughly represent the relative proportions of middle- and longwave cones in the normal retina, then the chance that 10 (non-shortwave) cones would be long-wave is $(69 / 118)^{10}$, i.e. 0.0047 or less than one in two hundred.

\section{ACKNOWLEDGEMENTS}

We are grateful to our patient for his skilful and careful observations, to M. Downham for experimental assistance, to $\mathrm{R}$. Hammans for preparation of figures, and to A. Stockman for guidance on computing. This work was supported by MRC Grant No. G 8206715 N.

\section{REFERENCES}

Albert, D. M. Ocular melanoma: a challenge to visual science. Investig. Ophthalmol. Vis. Sci. 23: 550-580 (1982).

Alpern, M. and Wake, T. Cone pigments in human deutan colour vision defects. J. Physiol., Lond. 266: 595-612 (1977).

Bowmaker, J. K., Dartnall, H. J. A. and Mollon, J. D. Microspectrophotometric demonstration of four classes of photoreceptor in an Old World primate, Macaca fascicularis. J. Physiol. 298: 131-143 (1980). 
Clerk Maxwell, J. On the theory of colours in relation to colour-blindness. In: Researches on Colour Blindness (Wilson, G., ed.). Su therland and Knox, Edinburgh (1855).

Committee on Vision, National Research Council (USA). Procedures for Testing Color Vision (Report of Working Group 41); National Academy Press, Washington (1981).

Dain, S. J. Assessment of the FM 100-Hue derivative tests. In: Colour Vision Deficiencies V (Verriest, G., ed.). Hilger, Bristol (1980).

Dartnall, H. J. A., Bowmaker, J. K. and Mollon, J. D. Human Visual Pigments: Microspectrophotometric Results from the Eyes of Seven Persons. Proc. Roy: Soc. B 220: $115-130$ (1983).

Ebrey, G. T. and Honig, B. New wavelength dependent visual pigment nomograms. Vision Res. 17: 147-151 (1977).

Farnsworth, D. Tritanomalous vision as a threshold function. Die Farbe 4: 185-197 (1955).

Hurvich, L. M. and Jameson, D. On the measurement of dichromatic neutral points. Acta Chromatica 2: 207-216 (1974).

Knowles, A. and Dartnall, H. J. A. The Photobiology of Vision. Volume 2A of Davson, H., ed. The Eye. Academic Press, New York (1977).

MacNichol, E. F., Jr., Levine, J. S., Mansfield, R. J. W., Lipetz and Collins, B. A. Microspectrophotometry of visual pigments in primate photoreceptors. In: Colour Vision: Physiology and Psychophysics (Mollon, J. D. and Sharpe, L. T., eds.), pp. 13-38. Academic Press, London (1983).

Mollon, J. D. A taxonomy of tritanopias. Doc. Ophthalmol. Proc. Series 33: 87-101 (1982).

Mollon, J. D. and Polden, P. G. An anomaly in the response of the eye to light of short wavelengths. Phil. Trans. Roy. Soc. B, 278, No. 960. (1977).

Rushton, W. A. H. A foveal pigment in the deuteranope. J. Physiol., Lond. 176: 24-37 (1965).

Voigt, J. H. Des Herrn Giros von Gentilly Muthmasungen über die Gesichtsfehler bey Untersuchung der Farben. Magazin für das Neueste aus der Physik und Naturgeschichte (Gotha) 1: 57-61 (1781).

Werner, J. S. and Wooten, B. R. Age changes in ocular media density and consequences for colour vision. In: Colour Vision Deficiencies V (Verriest, G., ed.) pp. 355359. Hilger, Bristol (1979).

Willmer, E. N. Further observations on the properties of the central fovea in colour-blind and normal subjects. J. Physiol. 110: 422-446 (1949).

Wright, W. D. The rays are not coloured, pp. 77-78 Hilger, Bristol (1967).

Wyszecki, G. and Stiles, W. S. Color Science. Wiley, New York (1982).

Author's address:

Dr J. D. Mollon

Department of Experimental Psychology

University of Cambridge

Downing Street

Cambridge CB2 3EB

U.K. 